Sādhanā Vol. 40, Part 3, May 2015, pp. 819-833. (C) Indian Academy of Sciences

\title{
Break-up of a non-Newtonian jet injected downwards in a Newtonian liquid
}

\author{
ABSAR M LAKDAWALA ${ }^{1}$, ROCHISH THAOKAR ${ }^{2}$ and \\ ATUL SHARMA ${ }^{1, *}$ \\ ${ }^{1}$ Department of Mechanical Engineering, Indian Institute of Technology Bombay, \\ Powai, Mumbai 400076, India \\ ${ }^{2}$ Department of Chemical Engineering, Indian Institute of Technology Bombay, \\ Powai, Mumbai 400076, India \\ e-mail: atulsharma@iitb.ac.in
}

MS received 9 May 2014; revised 23 December 2014; accepted 8 January 2015

\begin{abstract}
The present work on downward injection of non-Newtonian jet is an extension of our recent work (Lakdawala et al, Int. J. Multiphase Flow. 59: 206-220, 2014) on upward injection of Newtonian jet. The non-Newtonian rheology of the jet is described by a Carreau type generalized Newtonian fluid (GNF) model, which is a phenomenological constitutive equation that accounts for both rate-thinning and ratethickening. Level set method based numerical study is done for Newtonian as well as various types of shear thinning and thickening jet fluid. Effect of average injection velocity $\left(V_{a v, i}\right)$ is studied at a constant Reynolds number $R e=14.15$, Weber number $W e=1$, Froude number $F r=0.25$, density ratio $\chi=0.001$ and viscosity ratio $\eta=0.01$. CFD analysis of the temporal variation of interface and jet length $\left(L_{j}\right)$ is done to propose different types of jet breakup regimes. At smaller, intermediate and larger values of $V_{a v, i}$, the regimes found are periodic uniform drop (P-UD), quasi-periodic non-uniform drop (QP-NUD) and no breakup (NB) regimes for a shear thinning jet; and periodic along with Satellite Drop $(\mathrm{P}+\mathrm{S})$, jetting $(\mathrm{J})$ and no breakup (NB) regimes for a shear thickening jet, respectively. This is presented as a drop-formation regime map. Shear thickening (thinning) is shown to produce long (short) jet length. Diameter of the primary drop increases and its frequency of release decreases, due to increase in stability of the jet for shear thickening as compared to thinning fluid.
\end{abstract}

Keywords. Generalized Newtonian fluid model; periodic drop formation; quasiperiodic drop formation; satellite drop formation, jetting, no breakup.

*For correspondence 


\section{Introduction}

Breakup of a jet or a drop into another immiscible liquid has been studied extensively, due to their industrial application such as liquid-liquid extraction and direct heat transfer. Mono-dispersed emulsion produced by the breakup of a jet under co-flowing external fluid (Cramer et al 2004) has diverse applications in chemical, pharmaceutical, food and cosmetic industries. A wide variety of applications involve formation of drops from a liquid jet leaving a tube in a pool of another immiscible liquid; for example, property measurement, combustion, atomization and spray coating, crop spraying, ink jet printing, printing of polymer transistors, and microarraying for genomics, combinatorial chemistry and drug discovery. The success of such cutting-edge technologies depends strongly on the development of accurate methods of computing the dynamics of drop formation. In many applications involving drops, and in particular ones used in printing and coating, the liquids encountered are non-Newtonian.

For breakup of jet of a non-Newtonian liquid, Cooper-White et al (2002) experimentally studied the dynamics of formation of drops of low viscosity elastic fluids of constant shear viscosity and compared their behavior to drops of Newtonian glycerol-water solutions. Breakup of a Viscoelastic jet was studied experimentally by Mun et al (1998) for the effects of polymer concentration and molecular weight; and by Christanti \& Walker $(2001,2002)$ for a jet subjected to natural and forced disturbances. Although there are numerous numerical results on the breakup of a jet of Newtonian fluid, virtually no such results are found for non-Newtonian fluid; except for the works by Yildırım \& Basaran (2006) and Homma et al (2007).

The present work on the injection of a non-Newtonian jet is in-continuation of our recent work (Lakdawala et al 2014) on injection of a Newtonian fluid; into another Newtonian fluid. Lakdawala et al (2014) presented a novel procedure - based upon physical interpretation of the various functions in the Level Set Method (Gada \& Sharma 2009) - to calculate certain parameters (diameter as well as frequency of drop formation and temporal variation of jet length at the axis), which characterize the unsteady interface-dynamics. This was used to propose three modes of drop formation: Periodic Uniform Drop formation (P-UD), Quasi-Periodic Non-Uniform Drop formation (QP-NUD) and Chaotic Non-Uniform Drop formation (C-NUD); for six different combinations of the dispersed and continuous fluid, subjected to various injection velocities. They also presented a drop formation regime map for various Weber number and viscosity ratios. Finally, they studied the effect of various regimes on the mean value of jet dynamics parameters (jet breakup length, detached drop diameter and drop formation frequency).

The objective of the present work is to explore the various types of flow regimes and their effect on the jet dynamics parameters in non-Newtonian fluid system. This was done for the injection of Newtonian fluid in our previous work (Lakdawala et al 2014). Moreover, a more commonly applied downward injection is considered in the present as compared to upward injection in the previous work. One such application corresponds to inkjet printing, with a downward injection of a non-Newtonian fluid.

\section{Physical description of the problem}

The system consists of a heavier non-Newtonian fluid 1 leaving the tip of a tube (of radius $r_{1}$ ) into a cylindrical tank (of length $l$ and radius $r_{2}$ ), filled-in with a stationary lighter Newtonian fluid 2, shown in figure 1. A heavier non-Newtonian/injected in a Newtonian/continuous fluid is encountered in an ink-jet printing application. It can be seen that the dispersed fluid is injected 


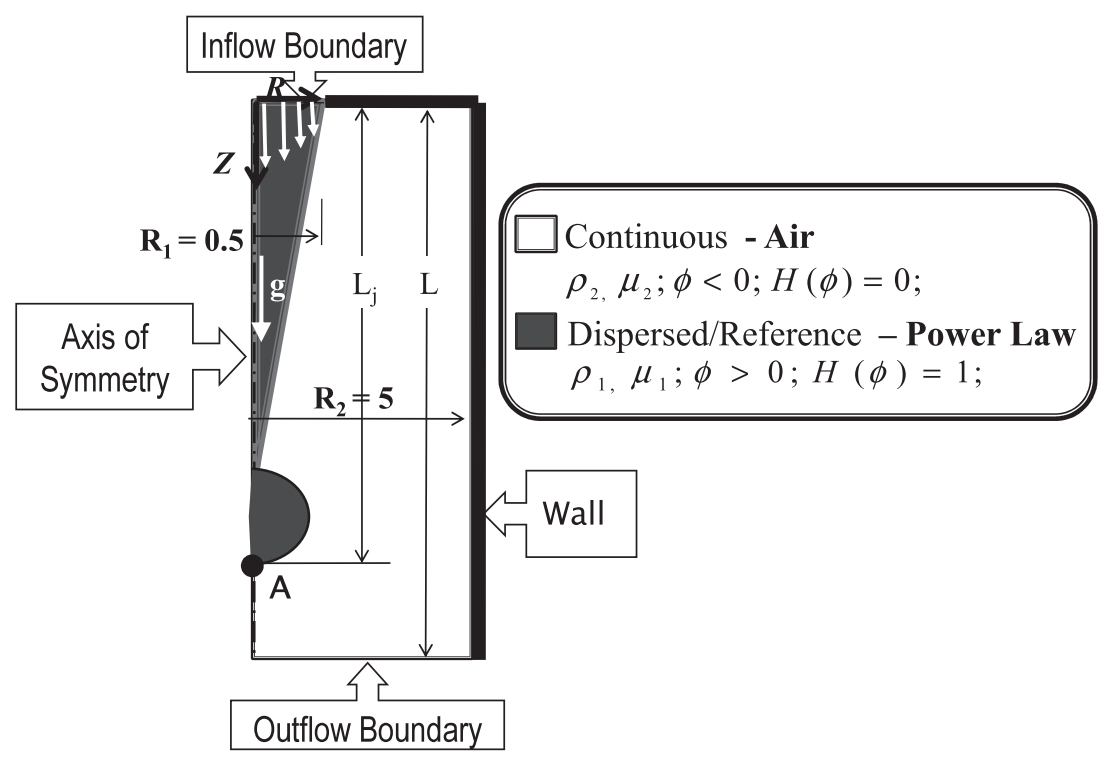

Figure 1. Computational domain and boundary conditions for an axi-symmetric non-Newtonian liquid jet injected into another immiscible Newtonian liquid.

downward with a fully developed axial velocity profile $V_{i}=2 V_{a v, i}\left(1-R^{2}\right)$. Both the fluids are incompressible and immiscible.

The figure shows an axi-symmetric computational domain and boundary conditions used in the present work. Note that the heavier/dispersed fluid 1 is taken as the reference fluid and the tube diameter $d_{1}$ is taken as the length scale. The non-dimensional length of the domain is taken as $R_{2} \equiv r_{2} / d_{1}=5$ in the radial and $L \equiv l / d_{1}=80$ in the axial direction.

\section{Mathematical formulation}

\subsection{Single field formulation}

In the present work, the Navier-Stokes equations are coupled with level set method (LSM; to model the interface, proposed by Sussman et al 1994) by invoking the single field formulation; wherein a single velocity and pressure field is defined for both the fluids. Homogeneous material properties are considered to be different for each phase, i.e., the bulk fluids are incompressible. Moreover, the surface tension force at interface is modeled as volumetric source term in the momentum equation; non-zero only at the interface. The surface tension coefficient is assumed to be constant and its tangential variation along the interface is neglected. It is assumed that the interface is thin and massless with no slip in tangential velocity. Recently, a comprehensive review on level set method was presented by Sharma (2015).

Physically relevant interface is of zero thickness and is represented in LSM by $\phi=0$; with $\phi>0$ in the dispersed and $\phi<0$ in the continuous fluid. However, a sharp change in thermophysical properties and surface-tension force across the interface leads to numerical instability. This is avoided by considering numerically relevant diffused interface of finite thickness $2 \epsilon$. The smeared interface is defined as $-\epsilon<\phi<\epsilon$, where $\epsilon$ is the half thickness of interface and is commonly taken as a factor of grid spacing with $2 \epsilon=3 \Delta R$ (Sussman \& Pukett 2000). 
Note that, the interface thickness is negligible on a fairly fine grid. Although origins of the LS method lie in mathematical sources, Gada \& Sharma (2009) proposed physical interpretation of various functions in LSM and used it for volume (mass) conservation law based derivation of continuity (level-set advection) equation. Non-dimensional governing equations for simulation of two-phase flow are given as

$$
\text { Volume conservation (continuity) equation: } \nabla \cdot \mathbf{U}=0
$$

$$
\text { Mass conservation (level-set advection) equation: } \frac{\partial \phi}{\partial \tau}+\mathbf{U} \cdot \nabla \phi=0
$$

Momentum conservation equation:

$$
\frac{\partial\left(\rho_{m} \mathbf{U}\right)}{\partial \tau}+\nabla \cdot\left(\rho_{m} \mathbf{U U}\right)=-\nabla P+\frac{1}{R e} \nabla \cdot\left(2 \mu_{m} D\right)-\frac{\rho_{m}}{F r^{2}} \hat{j}+\frac{1}{W e} \kappa \hat{n} \delta_{\epsilon}(\phi),
$$

where $\hat{j}$ is unit vector and the axial direction. 2D axi-symmetric form of above equations is solved using the boundary conditions; shown in figure 1. For the above equations, the nondimensional variables are expressed as

$$
\mathbf{U}=\frac{u}{v_{c}}, R=\frac{r}{d_{1}}, Z=\frac{z}{d_{1}}, \tau=\frac{t v_{c}}{d_{1}}, P=\frac{p}{\rho_{1} v_{c}^{2}}
$$

where capillary velocity $v_{c}=\sqrt{\sigma / \rho_{1} d_{1}}$ is the characteristic velocity scale and $\sigma$ is interfacial surface tension. Furthermore, Reynolds number $(R e)$, Froude number $(F r)$, Weber number $(W e)$ and non-dimensional injection velocity $\left(V_{a v, i}\right)$ are the non-dimensional governing parameters (based on properties of non-Newtonian jet fluid); defined as

$$
R e=\frac{\rho_{1} v_{c} d_{1}}{\mu_{1,0}}, F r=\frac{v_{c}}{\sqrt{g d_{1}}}, W e=\frac{\rho_{1} v_{c}^{2} d_{1}}{\sigma} \text { and } V_{a v, i}=\frac{v_{a v, i}}{v_{c}}
$$

where $v_{a v, i}$ is average injection velocity of jet fluid.

In the momentum equation, rate of deformation tensor, $D=\frac{1}{2}\left(\nabla \mathbf{U}+(\nabla \mathbf{U})^{T}\right)$. Furthermore, $\rho_{m}$ and $\mu_{m}$ are the mean non-dimensional density and viscosity, respectively. They are given as $\rho_{m}=H_{\epsilon}(\phi)+\chi\left(1-H_{\epsilon}(\phi)\right)$ and $\mu_{m}=\mu_{1} H(\phi)+\eta\left(1-H_{\epsilon}(\phi)\right)$, where the property ratio is given as

$$
\chi=\frac{\rho_{2}}{\rho_{1}} \text { and } \eta=\frac{\mu_{2}}{\mu_{1,0}}
$$

Furthermore, the smoothened Heaviside function is given as $H_{\epsilon}(\phi)=1$ if $\phi>\epsilon, H_{\epsilon}(\phi)=$ $\frac{\phi+\epsilon}{2 \epsilon}+\frac{1}{2 \pi} \sin \left(\frac{\pi \phi}{\epsilon}\right)$ if $|\phi| \leq \epsilon$ and $H_{\epsilon}(\phi)=0$ if $\phi<-\epsilon$. Moreover, in the momentum equation, $\hat{n}=\nabla \phi /|\nabla \phi|$ and $\kappa=-\nabla \cdot \hat{n}$ are the interface unit normal vector and curvature, respectively. Finally, $\delta_{\epsilon}(\phi)$ is smoothened Dirac delta function as $\delta_{\epsilon}(\phi)=\frac{\partial H_{\epsilon}(\phi)}{\partial \phi}=\frac{1}{2 \epsilon}+\frac{1}{2 \epsilon} \cos \left(\frac{\pi \phi}{\epsilon}\right)$ if $|\phi|<\epsilon$ otherwise $\delta_{\epsilon}(\phi)=0$.

The field of level set function obtained after solving the level-set advection equation does not remain a normal distance function field. It is necessary to maintain the constant width of the diffused interface, for accurate calculation of the fluid as well as thermo-physical properties and non-zero force at the interface. This is ensured by reinitializing the advected level set function field to signed normal distance function field; without altering the location of interface obtained after advection step. In the present work, a constraint-based PDE reinitialization procedure of Sussman et al (1998) is used. 


\subsection{Modeling of non-Newtonian rheology of the dispersed fluid}

Here, a generalized Newtonian fluid (GNF) model is used to describe the non-Newtonian rheology of the jet liquid. The model is a phenomenological constitutive equation that accounts for both rate thinning and rate thickening (Song \& Xia 1994). It provides an explicit equation for the apparent viscosity function, given as

$$
\begin{aligned}
\mu_{1}=\operatorname{Gr} T r & \\
\text { where } & G r=(1-\beta)\left[1+\left(\alpha \dot{\gamma_{2}}\right)^{a_{1}}\right]^{\left((n-1) / a_{1}\right)}+\beta \\
& \operatorname{Tr}=\left(1-\operatorname{Tr}_{\infty}\right)\left[1+\left(\lambda\left|\dot{\gamma_{3}}\right|\right)^{a_{2}}\right]^{\left((m-1) / a_{2}\right)}+\operatorname{Tr}_{\infty},
\end{aligned}
$$

where $G r$ is rate thinning and $T r$ is rate thickening function; expressed above as three parameter Carreau type equations. Furthermore, $\dot{\gamma}_{2}$ is second and $\dot{\gamma}_{3}$ is third invariants of the rate of deformation tensor; and $\alpha$ and $\lambda$ are the inverse of characteristic deformation rates. Also $0<\beta \leq 1$ for rate thinning and $1 \leq \operatorname{Tr}_{\infty}$ for rate thickening effects are dimensionless infinite deformation rate viscosity; non-dimensionalized using zero shear viscosity, i.e., $\beta \& T r_{\infty}=\mu_{1, \infty} / \mu_{1,0}$. Finally, $n \leq 1$ and $m \leq 1$ are the power law constants for the rate thinning and thickening,

respectively. Thus, $\lim _{\gamma_{2}, \dot{\gamma}_{3} \rightarrow 0} \mu_{1}=1$ and $\lim _{\gamma_{2}, \dot{\gamma}_{3} \rightarrow \infty} \mu_{1}=\beta T r$. The two invariants of the rate of deformation tensor are given - in cylindrical coordinate - as

$$
\begin{aligned}
& \dot{\gamma}_{2}=2\left(\frac{\partial U}{\partial R}\right)^{2}+\left(\frac{\partial U}{\partial Z}+\frac{\partial V}{\partial R}\right)^{2}+2\left(\frac{\partial V}{\partial Z}\right)^{2}+2\left(\frac{U}{R}\right)^{2} \\
& \dot{\gamma}_{3}=2 \frac{U}{R}\left[4 \frac{\partial U}{\partial R} \frac{\partial V}{\partial Z}-\left(\frac{\partial V}{\partial R}+\frac{\partial U}{\partial Z}\right)^{2}\right],
\end{aligned}
$$

where $U$ is the radial and $V$ is the axial components of the velocity. Here, $a_{1}$ and $a_{2}$ are taken as 2 and 3, respectively; however, different values are taken for code validation.

\section{Numerical methodology}

In the present work, an in-house code based on a novel Dual Grid Level Set Method (DGLSM; Gada \& Sharma 2011) in 2D axi-symmetric cylindrical coordinate system - developed by Gada (2012) - is used. The governing equations are discretized on a Cartesian MAC-type staggered grid arrangement, to avoid pressure velocity decoupling. A Finite Volume Method (FVM) based semi-explicit pressure projection method is used to solve the Navier-Stokes equations. The continuity and the diffusion term in the momentum equation are treated implicitly; whereas, the advection and all body forces in momentum equation are treated explicitly. The advection and diffusion terms in momentum equation are discretized using 2nd order TVD Lin-Lin and central difference scheme, respectively. LS advection equation - discretized by finite difference method - is solved explicitly with Runge-Kutta and WENO scheme for temporal and spatial terms, respectively. The reinitialization equation is solved using ENO scheme.

The viscosity field in the domain is calculated using Eq. 6 and Eq. 7. Note that velocity field of previous time-instant is used to calculate two invariants of the rate of deformation tensor in Eq. 7. Furthermore, the time step is calculated based on CFL, grid Fourier number and capillary time step restriction. The density, viscosity and mass flux are calculated at LS nodes first, and their values are interpolated to required locations. 

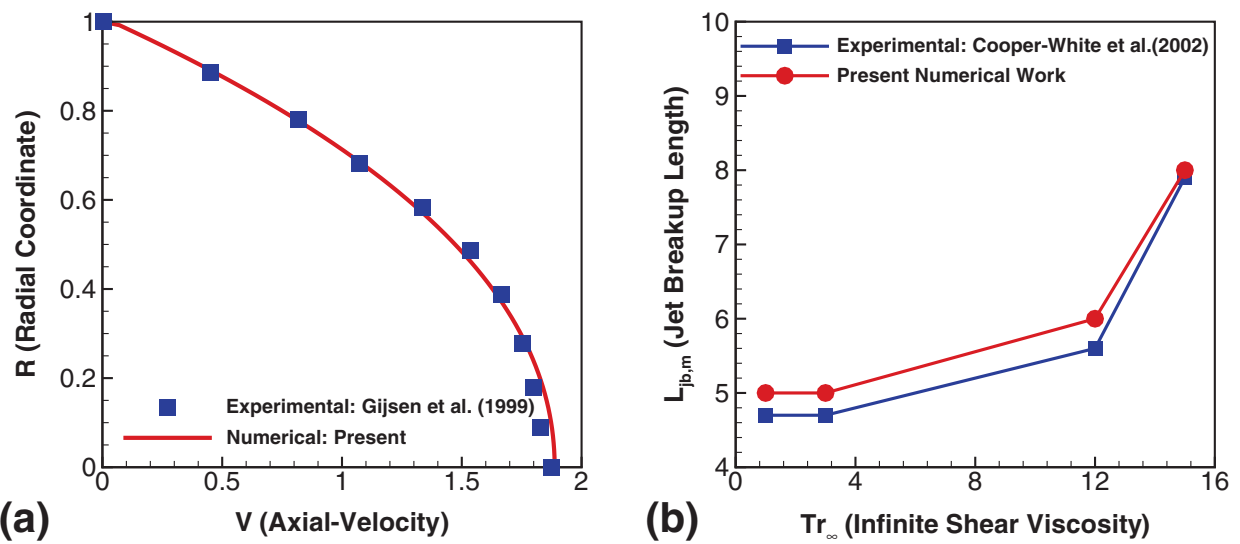

Figure 2. Comparison of published experimental and present numerical results for (a) non-dimensional axial-velocity profile for fully developed non-Newtonian flow in a pipe and (b) variation of jet break-up length with increasing infinite shear viscosity.

The present simulations are done on a grid size of $100 \times 800$ and a time step of 0.0001 . Grid independence and code validation study - of the present LSM based code - for the injection of Newtonian liquid jet is presented in our recent work (Lakdawala et al 2014). However, in order to validate the implementation of the non-Newtonian rheology by the generalized Newtonian fluid (GNF) model, a single phase flow - corresponding to developing non-Newtonian fluid flow in a pipe - and the present two-phase flow are taken as the benchmark problems. Figure 2(a) shows an excellent agreement between the published experimental (Gijsen et al 1999) and present numerical results, for fully developed dimensionless axial velocity profile at $\mathrm{Re}=36$. The results correspond to a blood analog fluid of concentrated solution of KSCN (potassium thio-cyanate) with aqueous Xanthan gum, which showed shear thinning behavior. Furthermore, non-Newtonian rheological parameters considered are $\beta=0.1, \alpha=0.11$, $n=0.392, a_{1}=0.644, T r_{\infty}=1, \lambda=1$, and $a_{2}=1$.

For the problem attempted here, good agreement between the present numerical and experimental results of Cooper-White et al (2002) is shown in figure 2(b); for variation of jet break-up length, with increasing infinite shear viscosity (corresponding to various shear thickening fluid). Cooper-White et al (2002) considered mixture of 50\% Glycerol and 50\% Water to obtain Newtonian fluid $\left(T r_{\infty}=1\right)$ and varied the concentration of polyethylene oxide (PEO) in the mixture of glycerol and water to obtain various shear thickening fluids; and considered air as the continuous fluid.

\section{Results and discussions}

A parametric study is done here for non-Newtonian shear thinning/thickening injecting fluid and compared with the results for Newtonian fluid. In this regard, the non-dimensional governing parameters $\left(V_{a v, i}, \alpha\right.$ and $\left.\lambda\right)$ are as follows:

Average injection velocity $\left(V_{a v, i}\right): 0.2$ to 0.6 in steps 0.1 .

Shear thinning: $\alpha=0.1,1 \& 10$ at $\beta=0.1, a_{1}=2, \operatorname{Tr}_{\infty}=1, a_{2}=1$ and $n=0.5$.

Shear thickening: $\lambda=0.1,1 \& 10$ at $\beta=1, a_{1}=2, \operatorname{Tr}_{\infty}=10, a_{2}=3$ and $m=0.5$.

Newtonian: $\beta=1$ and $\operatorname{Tr}_{\infty}=1$. 
Thus, a total of thirty-five 2D transient simulations are carried out to study the effect of nonNewtonian rheology and injection velocity on flow transition and drop-dynamics parameters. For any combination of fluid in the two-fluid system here, the velocity scale $\left(v_{c}=\sqrt{\sigma / \rho_{1} d_{1}}\right)$ is taken such that $W e=1$; whereas $R e, F r, \chi$ and $\eta$ will be a constant value (refer Eqs. 4 and 5) for a particular fluid combination. The constant values are taken here as $R e=14.15, F r=0.25$, $\chi=0.001$ and $\eta=0.01$.

\subsection{Interface-dynamics of non-Newtonian jet break-up}

For Newtonian jet break-up, Lakdawala et al (2014) proposed a novel method to characterize interface dynamics; also used here for non-Newtonian jet break-up. They defined jet length $L_{j}$ as the axial distance along the axis after which there is first change from dispersed to continuous fluid. This is shown in figure 1 as the axial distance of a critical point $A$ from the inlet. It was computed as the minimum length along the axis - from the inlet - where the level set changes its sign (from positive to negative in figure 1). They discussed that a sudden decrease in the time-signal of $L_{j}$ indicates a breakup of the jet into a drop; and correlated the magnitude of the decrease with the size of the detached drop. They proposed an inverse of the time period between the two consecutive breakup as the frequency of drop formation (non-dimensional form called as Strouhal number $S t$ ); and used the value of Heaviside function to compute the diameter of the detached drop $\left(D_{d}\right)$.

Furthermore, using the temporal variation of $L_{j}$, Lakdawala et al (2014) identified three drop formation regimes: Periodic Uniform Drop formation (P-UD), Quasi-Periodic Non-Uniform Drop formation (QP-NUD) and Chaotic Non-Uniform Drop formation (C-NUD). Their P-UD regime corresponds to a periodic break-up of almost the same size of drop and QP-NUD regime to a periodic break-up of a drop of a particular size followed by another of a different size. In the QP-NUD regime, they proposed two frequencies: primary and secondary; corresponding to the frequency of formation of drop of any size for primary and of same size for secondary frequency.

In case of a non-Newtonian jet, in addition to the P-UD and QP-NUD regimes, Periodic drop formation with Satellite Drop $(\mathrm{P}+\mathrm{S})$, Jetting $(\mathrm{J})$ and No-Breakup $(\mathrm{NB})$ regimes are also found when the jet is injected downward into a Newtonian liquid. Temporal variation of instantaneous interface and jet length - at various values of average injection velocity - is shown in figure 3 for shear-thinning fluid at $\alpha=1$ and in figure 4 for shear-thickening fluid at $\lambda=10$. The figures also show color contour of mean viscosity of the non-Newtonian fluids; inside the jet/drop.

5.1a Shear thinning fluid: Figure 3 shows a considerable change in the drop dynamics with increasing $V_{a v, i}$, caused by the increase in the inertia of the shear thinning fluid $(\alpha=1)$ at the inlet. The figure shows that the volume of the incoming jet increases with the continuous downward injection of the shear-thinning liquid. Once the jet volume becomes sufficiently large, the gravity as compared to surface tension force acting on the jet becomes large enough for the formation of neck on the jet and subsequently it breaks into drop. The successive pinch-off of the drop is self-similar. It can also be seen that the mean viscosity decreases $\left(\mu_{m}<1\right)$ during the formation of neck - before the break-up of a jet into a drop - due to an increase in the shear rate.

The general pattern of the time signal of jet length (figure $3 a_{9}$ ) is its gradual increase up to a certain length, followed by a sharp decay due to detachment of a drop, with a subsequent repeated buildup in length to form the next drop which results in a "sawtooth" pattern. The nondimensional period of drop formation can be found as the time period between successive crests, while the vertical distance between the crest and trough can roughly correlate with the volume of the detached drop (Lakdawala et al 2014). 


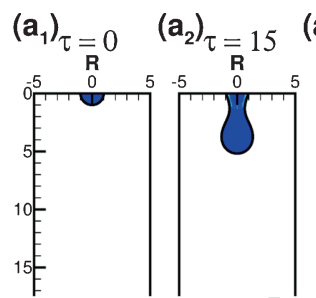

$\left(\mathrm{a}_{3}\right)_{\tau=20}$

$\left(\mathbf{a}_{4}\right)_{\tau=30}$

$\left(a_{5}\right)_{\tau=35}$

$\left(a_{6}\right)_{\tau=45}$

$\left(a_{7}\right)_{\tau=50}$

$\left(a_{8}\right)_{\tau=65}$

$\left(a_{9}\right)$
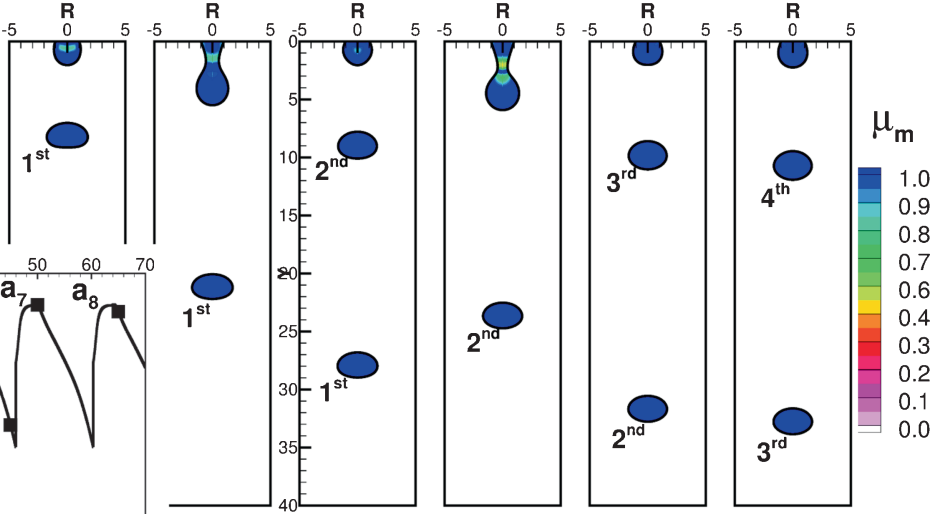

$\left(\mathbf{b}_{1}\right)_{\tau=0}$

$\left(\mathbf{b}_{2}\right)_{\tau=15}$

$\left(b_{3}\right)_{\tau=25}$

$\left(\mathbf{b}_{4}\right)_{\tau=35}$

$\left(b_{5}\right)_{\tau=40}$

$\left(\mathbf{b}_{6}\right)_{\tau=50}$

$\left(b_{7}\right)_{\tau=60}$

$\left(b_{8}\right)_{\tau}=70$

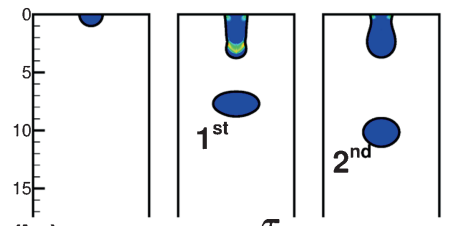

$\left(b_{9}\right)$
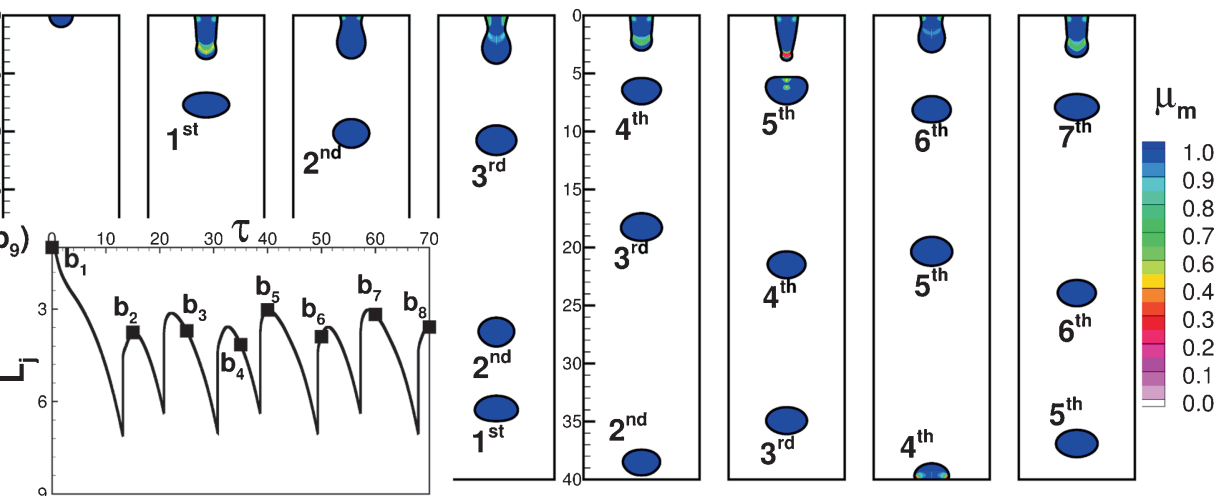

$\left(\mathbf{c}_{1}\right)_{\tau=0}$

$\left(\mathbf{c}_{2}\right)_{\tau=10}$

$\left(\mathbf{c}_{3}\right)_{\tau=15}$

$\left(\mathbf{c}_{4}\right)_{\tau=20}$

$\left(c_{5}\right)_{\tau=40}$

${ }^{\left(\mathbf{c}_{6}\right)} \tau=50$

$\left(c_{7}\right) \tau=60$

${ }^{\left(c_{8}\right)} \tau=70$
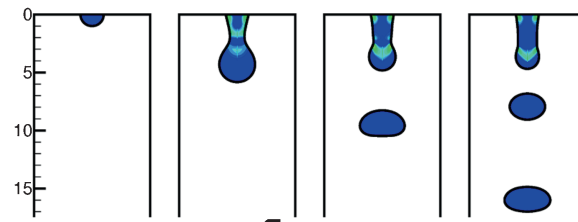

(c)
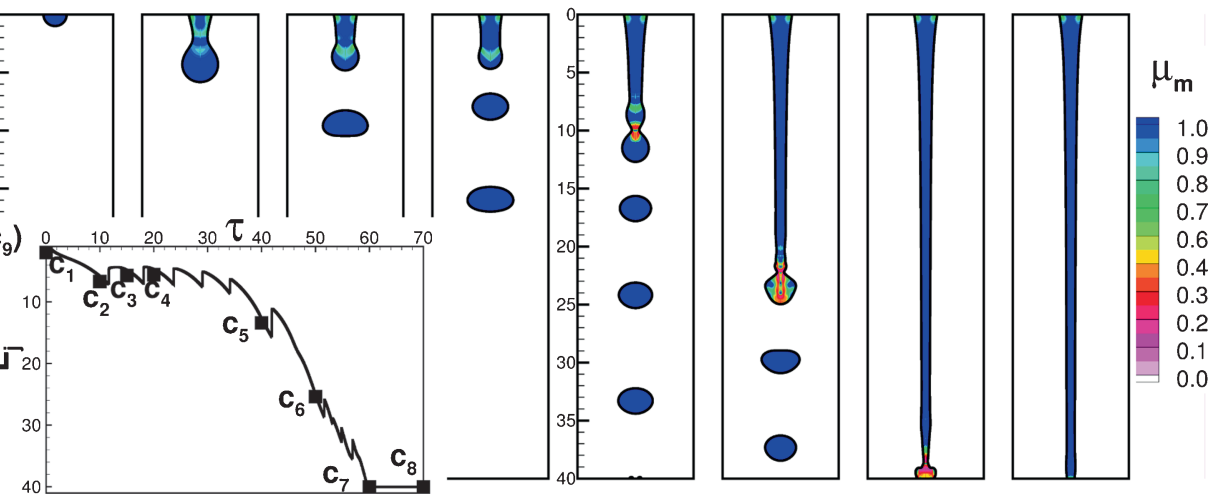

Figure 3. Temporal variation of instantaneous interface, contour of mean viscosity and jet length $\left(L_{j}\right)$ for shear thinning fluid $(\alpha=1)$, at an average injection velocity of $\left(a_{1}-a_{9}\right) 0.3,\left(b_{1}-b_{9}\right) 0.5$ and $\left(c_{1}-c_{9}\right)$ 0.6 ; indicating P-UD, QP-NUD and NB regime respectively. Sub-figure $\left(a_{9}\right)$ represents temporal variation of $L_{j}$, with the symbols corresponding to the time instant for the instantaneous plots in $\left(a_{1}\right)-\left(a_{8}\right)$; similarly for subfigures $\left(b_{9}\right)$ and $\left(c_{9}\right)$. 

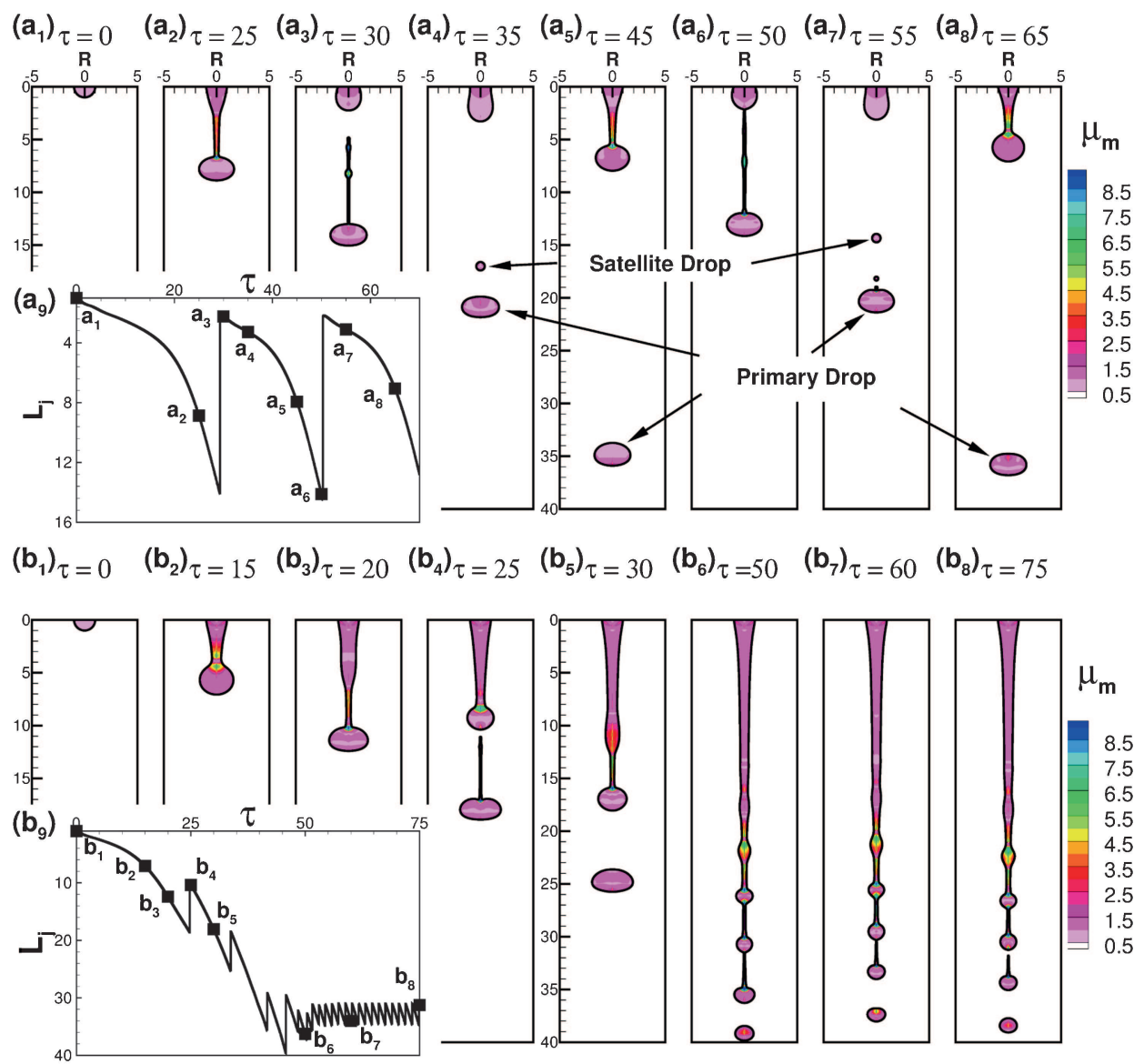

$\left(\mathbf{c}_{1}\right)_{\tau}=0$

$\left(\mathbf{c}_{2}\right)_{\tau=10} \quad\left(\mathbf{c}_{3}\right)_{\tau=20}$

$\left(\mathbf{c}_{4}\right)_{\tau=25}$

$\left(c_{5}\right)_{\tau}=30$

$\left(\mathbf{c}_{6}\right)_{\tau=45}$

$\left(c_{7}\right)_{\tau=50}$

$\left.\mathbf{c}_{8}\right)_{\tau=60}$

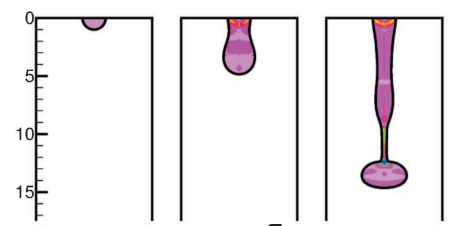

$\left(\mathbf{c}_{9}\right)$
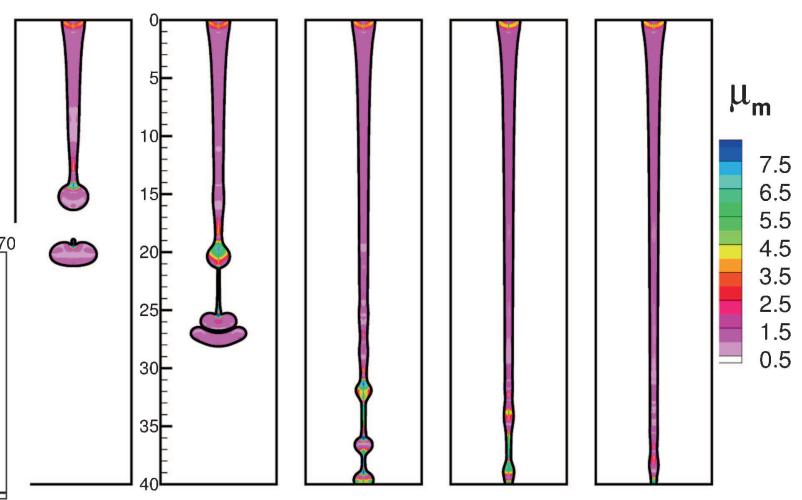

Figure 4. Temporal variation of instantaneous interface, contour of mean viscosity and jet length $\left(L_{j}\right)$ for shear thickening fluid $(\lambda=10)$, at an average injection velocity of $\left(a_{1}-a_{8}\right) 0.2,\left(b_{1}-b_{8}\right) 0.4$ and $\left(c_{1}-c_{8}\right)$ 0.5 ; indicating $\mathrm{P}+\mathrm{S}, \mathrm{J}$ and NB regime respectively. Sub-figure $\left(a_{9}\right)$ represents temporal variation of $L_{j}$, with the symbols corresponding to the time instant for the instantaneous plots in $\left(a_{1}\right)-\left(a_{8}\right)$; similarly for subfigures $\left(b_{9}\right)$ and $\left(c_{9}\right)$. 
For $V_{a v, i}=0.3$, figure $3 a_{1}-3 a_{9}$ shows that the temporal variation of jet length is periodic (compare time period between the successive crests in figure $3 a_{9}$ ), with the formation of drop of the same size (average diameter $D_{a v}=2.87$ ); indicating drop formation regime as P-UD. For $V_{a v, i}=0.5$, figure $3 b_{1}-3 b_{8}$ shows formation of periodically repeating bigger $\left(1^{\mathrm{st}}, 3^{\mathrm{rd}}, 5^{\text {th }}\right.$ and $\left.7^{\text {th }}\right)\left(D_{a v}=3.6\right)$ and smaller $\left(2^{\text {nd }}, 4^{\text {th }}\right.$ and $\left.6^{\text {th }}\right)\left(D_{a v}=2.48\right)$ drops. Figures $3 a_{9}$ and $3 b_{9}$ show a periodic and quasi-periodic variation in the temporal variation in the jet length, respectively. This indicates that the respective drop formation regime is P-UD and QP-NUD. At higher non-dimensional injection velocity $\left(V_{a v, i}=0.6\right)$, figure $3 c_{1}-3 c_{8}$ shows a break-up of injected non-Newtonian fluid into droplets up to $\tau=40$. Thereafter, the larger injection velocity results in a larger downward velocity for the jet than for the droplets. Thus, the jet merges with the droplets and results in a continuous jet (figure $3 c_{7}$ ).

5.1b Shear thickening fluid: Figure 4 shows a jet break-up for a shear thickening fluid. The mean viscosity is found to increase during the formation of neck; before the break-up of a jet into a drop. For $V_{a v, i}=0.2$, after the initial breakup, figure $4 a_{1}-4 a_{8}$ shows recoiling of a thread due to the unbalanced force of surface tension. After the initial pinch-off, there is a thin thread-like interface hanging between the primary drop and the orifice (figure $4 a_{3}$ ). The newly freed end of the thread recoils due to the unbalanced force of the surface tension. Meanwhile, the primary drop tends to become spherical because of the capillary force. A large curvature, therefore, develops at the joining point of the primary drop and the thread; leading to a large capillary pressure and break-up of the thread. As a result of the thread rupturing at both ends sequentially, a drop of much smaller size - called as satellite drop - is formed in-between the primary drop and the cone-shaped liquid remaining on the orifice (figure $4 a_{4}$ ). This drop formation regime is called as $\mathrm{P}+\mathrm{S}$.

All the cases of the drop of the shear-thickening liquid investigated here show long necks/threads which exhibit bead-on-string patterns prior to pinch-off. Similar results were reported, for capillary breakup of rate-thickening liquids, by Entov \& Shmaryan (1997). In these cases, the neck breaks at multiple locations to produce satellites. However, such patterns have not been observed with shear thinning or Newtonian jet; and should be used in applications where satellite formation is undesirable.

For $V_{a v, i}=0.4$, figure $4 b_{1}-4 b_{9}$ shows generation of smaller drop at a very high frequency, with a very large breakup length; indicating the jetting $(\mathrm{J})$ regime. Whereas, for $V_{a v, i}=0.5$, figure $4 c_{1}-4 c_{9}$ does not show the break up; NB regime.

\subsection{Drop formation regime map}

With increasing injection velocity of Newtonian and non-Newtonian fluid, a flow regime map representing the various regimes of drop formation (P-UD, QP-NUD, P $+\mathrm{S}, \mathrm{J}$ and NB) are shown in figure 5 . With increasing $V_{a v, i}$, the figure shows a transition in the drop formation regime from P-UD to QP-NUD to NB regime, for shear-thinning as well as Newtonian fluid; except at $\alpha=10$ where the transition to NB regime is not found up to $V_{a v, i}=0.6$ studied here. Whereas for a shear thickening fluid, the transition is seen from $\mathrm{P}+\mathrm{S}$ to $\mathrm{J}$ to $\mathrm{NB}$ regime at $\lambda=10$ and directly from $\mathrm{P}+\mathrm{S}$ to $\mathrm{NB}$ regime at $\lambda=0.1$ and 1 . At low injection velocity, periodic drop formation with satellite $(\mathrm{P}+\mathrm{S})$ for shear thickening fluid is primarily due to recoiling of thread after initial pinch-off.

For shear thickening fluid, transition from periodic drop formation to jetting and no breakup occurs due to stability provided by higher viscosity at $\lambda=10$; vice-versa for shear thinning fluid at $\alpha=10$, where quasi-periodic drop formation regime continues up to the largest $V_{a v, i}$. 


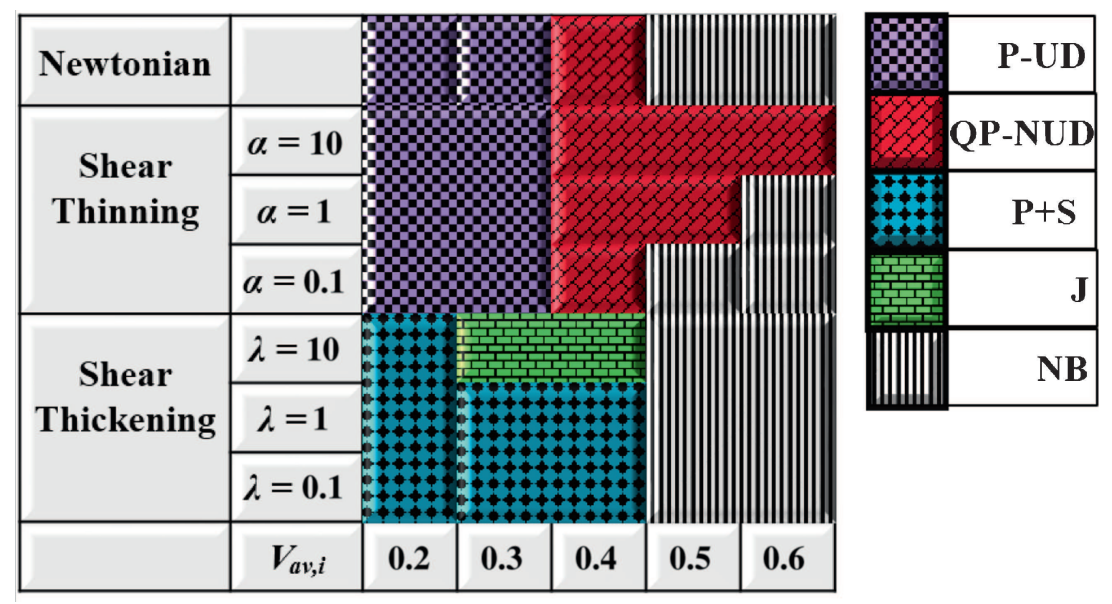

Figure 5. Newtonian and non-Newtonian (shear thinning and thickening) jet break-up regime map, at various values of average injection velocity. Drop formation regimes: periodic uniform drop (P-UD), quasiperiodic non-uniform drop (QP-NUD), periodic with satellite drop $(\mathrm{P}+\mathrm{S})$, jetting $(\mathrm{J})$ and no break-up (NB).

\subsection{Jet dynamics parameters}

Statistically steady time-averaged/mean value of non-dimensional drop-dynamics parameters such as jet breakup length $L_{j b, m}\left(L_{j}\right.$ at the break-up of a drop), drop diameter $D_{d, m}$ and frequency of drop formation $S t_{m}$ are computed from the sufficiently long duration unsteady results; computed using a level set based novel procedure, presented by Lakdawala et al (2014). With increasing average injection velocity, a variation of these parameters in the different regime of drop formation is shown in figure 6, for the Newtonian and various types of non-Newtonian jet. The parameters are not relevant and are not shown in the figure for $\mathrm{J}$ and NB regime.

With increasing $V_{a v, i}$, figure 6 shows a monotonic increase in $L_{j b, m}, D_{d, m}$ and $S t_{m}$; except a slight decrease in $L_{j b, m}$ and $D_{d, m}$ at larger values of $V_{a v, i}$. The slight decrease may be due to a transition from P-UD to QP-NUD resulting in the formation of non-uniform drop, for the shear thinning jet with $\alpha=10$ (figure 5). Note that the increase in figure 6 is marginal for $L_{j b, m}$ and $D_{d, m}$; and substantial for $S t_{m}$.

The figure also shows the effect of non-Newtonian rheology on drop dynamics parameters. Figure 6a shows that $L_{j b, m}$ decreases slightly for shear-thinning jet, with increasing $\alpha$; and increases substantially for shear thickening jet, with increasing $\lambda$. Theoretically, surface tension force has to move fluid particles of the jet to allow the surface tension driven instability to grow (Eggers \& Villermaux 2008). According to similarity solution of Lister \& Stone (1998), the internal viscous stresses of the jet is associated with extension of the thread. The viscous "brake" slows the growth of instability down. Also, according to the linear stability theory for an infinite liquid column suspended in another immiscible fluid (Tomotika 1935), the growth rate of disturbances decreases with increasing viscosity of the jet fluid. Hence, the jet is stabilized by a higher viscosity of shear thickening fluid and hence larger jet breakup length is observed as compared to shear thinning jet. Figure $6 b-c$ shows that a slight variation in diameter and frequency of drop formation with increasing $\alpha$ for shear-thinning and $\lambda$ for shear-thickening jet.

Note that the inverse of $\alpha(\lambda)$ indicates the shear rate at which shear thinning (thickening) starts. Hence, higher value of $\alpha(\lambda)$ means more shear thinning (thickening) effect which in turn decreases (increases) the viscosity of jet fluid. Furthermore, the high viscosity of the jet 

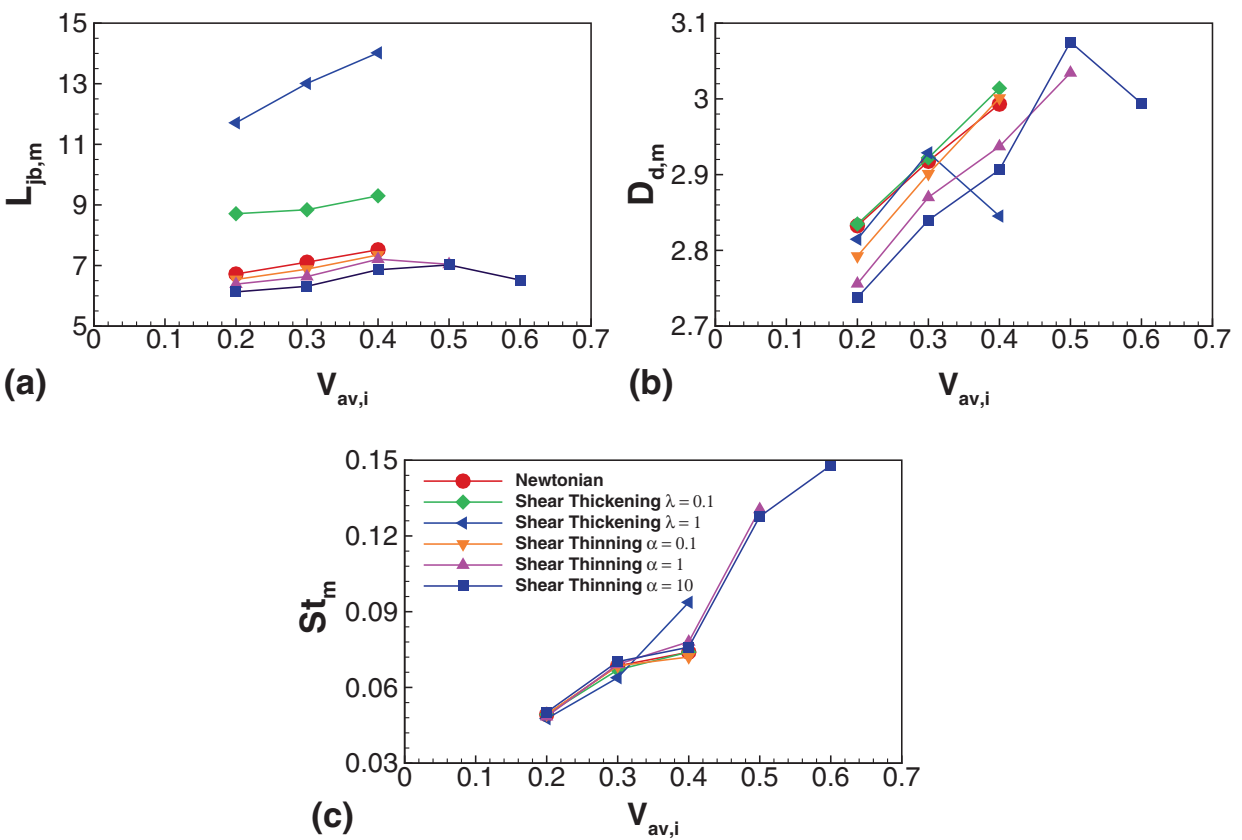

Figure 6. Effect of the constitutive model on mean non-dimensional values of (a) jet breakup length, (b) diameter of detached drop and (c) frequency of drop formation. Note that the legend in (c) is common for all the sub-figures.

fluid damps out the instability created by the surface tension, delaying the jet breakup and drop production. Thus, the jet breakup length for shear thickening jet is found to be larger as compared to that of shear thinning jet (figure 6a). As a result of the increased extension of the thread (for shear thickening), a longer elapsed time is required for the drop breakup. Due to longer elapsed time, the axial momentum of the liquid leaving the nozzle forces more liquid to squeeze into the drop; and causes the diameter of the detaching primary drop to increase for shear thickening jet as compared to that of shear thinning jet (figure 6b). Moreover, the damping effect decreases from shear thickening fluid to shear thinning fluid; thus, the frequency of the drop formation is higher for shear thinning fluid as compared to that of shear thickening fluid (see figure $6 \mathrm{c}$ ).

\section{Analysis of flow-pattern for the shear thinning and thickening jet fluid}

For the same value of average injection velocity of $V_{a v, i}=0.2$, figure 7 shows velocity distribution and contour of mean viscosity inside the non-Newtonian jet fluid before it breaks into periodic uniform drop (P-UD) for shear thinning $(\alpha=10)$ and periodic along with satellite drop $(\mathrm{P}+\mathrm{S})$ for shear thickening $(\lambda=10)$ jet fluid. Figure $7(\mathrm{a}, \mathrm{c})$ shows that the jet length for shear thickening as compared to thinning jet fluid is longer, with a substantially longer neck for the shear thickening fluid. However, for both the types of jet fluid, a vortex is seen on the either side of the jet which are of opposite sign; caused by the entrainment of the continuous fluid during the growth/elongation of jet. The entrainment seems to be more strong for the shear thickening as compared to thinning jet. Furthermore, the gradient of axial velocity inside the jet seems to be more stronger inside the shear thickening jet; indicated by a much larger change in the mean viscosity in figure 7(c) as compared to figure 7(a). 

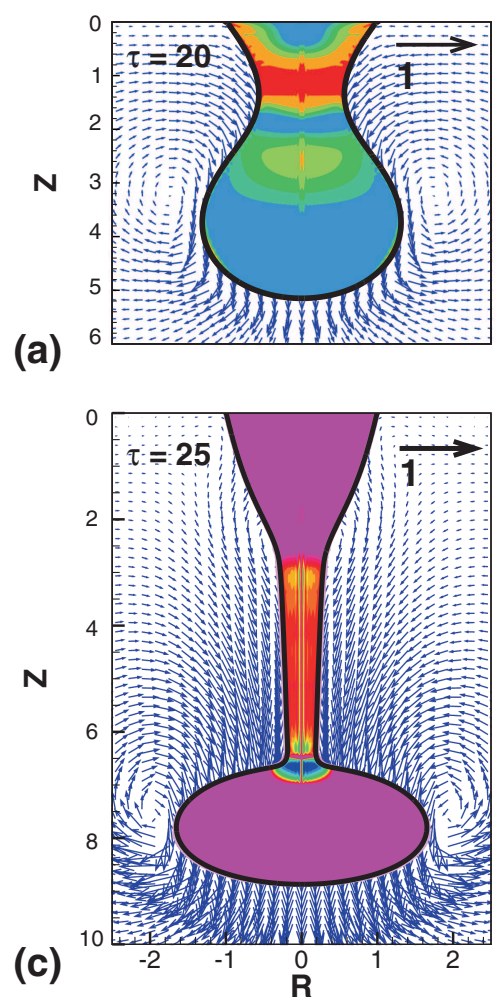
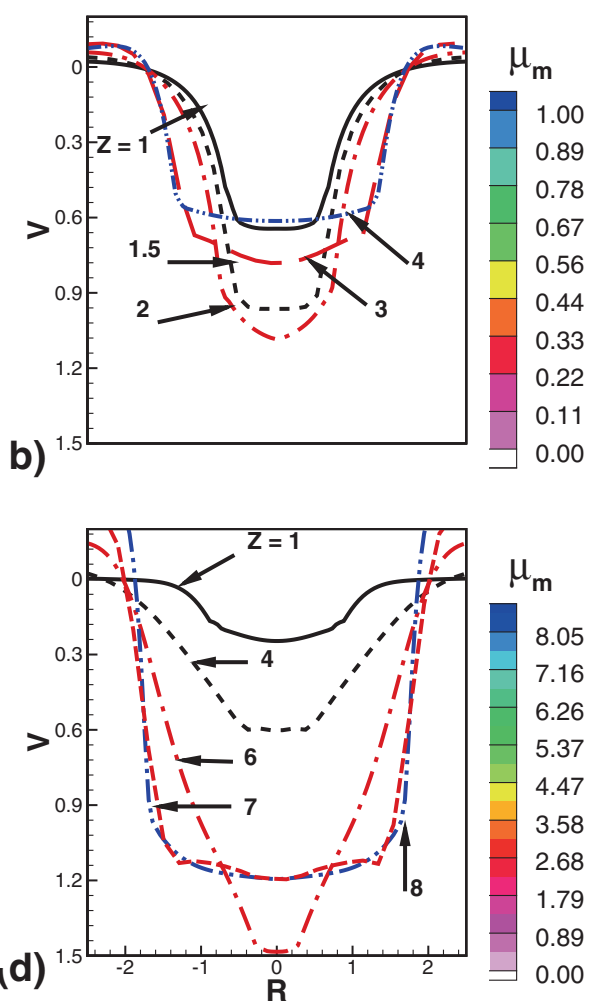

Figure 7. Shear (a,b) thinning $(\alpha=10)$ and $(\mathrm{c}, \mathrm{d})$ thickening $(\lambda=10)$ jet at $V_{a v, i}=0.2$ : $(\mathrm{a}, \mathrm{c})$ instantaneous interface, velocity vector (in the Newtonian continuous fluid) and contour of mean viscosity (in the non-Newtonian jet fluid); and (b,d) axial velocity profile at various axial locations.

For shear thinning fluid with $\alpha=10$, with increasing axial distance, figure 7(b) shows that the maximum in the axial velocity profile (seen at the axis of jet) increases up to the minimum cross-section (neck formation around $Z=2$ ) of the jet and then decreases. This is also seen in figure 7(d) for shear thickening fluid with neck formation around $Z=6$. However, the gradient of axial velocity along the axial direction seems to be much larger for the shear thickening as compared to thinning fluid. The larger velocity gradient and longer neck leads to formation of thread-like interface which generates a satellite along with a primary drop for the shearthickening fluid.

\section{Conclusions}

- Carreau type generalized Newtonian fluid (GNF) model is implemented in an in-house axisymmetric Dual Grid Level Set Method based code, to model non-Newtonian rheology of jet fluid.

- Formation of jet of non-Newtonian liquid and its subsequent breakup into drops is studied by changing the time constant $-\alpha$ and $\lambda$ for shear thinning and shear thickening fluid respectively - of GNF model. 
- Time signal analysis of jet length $\left(L_{j}\right)$ - at various average injection velocity $V_{a v, i}$ and rheological parameters ( $\alpha$ and $\lambda$ ) - is used to propose different jet breakup regimes such as Periodic uniform-drop formation (P-UD), Periodic drop formation with satellite drop (P $+\mathrm{S}$ ), Quasi-periodic non-uniform drop formation (QP-NUD), Jetting (J) and No breakup (NB). P, QP and NB regimes for shear thinning jet whereas $\mathrm{P}+\mathrm{S}$, JET and NB regimes for shear thickening jet are obtained at smaller, intermediate and larger values of $V_{a v, i}$, respectively. This is presented as a drop-formation regime map.

- A shear thickening jet results in a long thread which eventually breaks to produce many satellite drops; whereas a shear thinning jet produces mono-dispersed drops (without satellite) with frequency higher than that of a shear thickening fluid.

\section{Acknowledgement}

The support received from the Institute of Technology, Nirma University - for sending the first author to carry out research at Indian Institute of Technology Bombay - is gratefully acknowledged. Discussions with Dr. Vinesh H. Gada are highly appreciated. We are also thankful to the anonymous reviewer for his comments, which have helped us to come up with a substantially improved manuscript.

\section{References}

Christanti Y and Walker L M 2001 Surface tension driven jet breakup of strain-hardening polymer solutions. J. Non-Newtonian Fluid Mech. 100: 9-26

Christanti Y and Walker L M 2002 Effect of fluid relaxation time of dilute polymer solutions on jet breakup due to a forced disturbance. J. Rheol. 46(3): 733-748

Cooper-White J J, Fagan J E, Tirtaadmadja V, Lester D R and Boger D V 2002 Drop formation dynamics of constant low-viscosity, elastic fluids. J. Non-Newtonian Fluid Mech. 106: 29-59

Cramer C, Fischer P and Windhab E J 2004 Drop formation in a co-flowing ambient fluid. Chem. Eng. Sci. 59: 3045-3058

Eggers J and Villermaux E 2008 Physics of liquid jets. Rep. Prog. Phys. 71: 036601

Entov V M and Shmaryan L E 1997 Numerical modeling of the capillary breakup of jets of polymeric liquids. Fluid Dyn 32(5): 696-703

Gada V H and Sharma A 2009 On derivation and physical-interpretation of level set method based equations for two-phase flow simulations. Numer. Heat Transfer B. 56: 307-322

Gada V H and Sharma A 2011 On novel dual-grid level-set method for two-phase flow simulation. Numer. Heat Transfer B. 59: 26-57

Gada V H 2012 A novel level-set based CMFD methodology in 2D/3D cartesian and cylindrical coordinates and its application for analysis of stratified flow and film boiling. Ph.D Thesis, IIT Bombay

Gijsen F J H, Van de Vosse F N and Janssen J D 1999 The influence of the non-Newtonian properties of blood on the flow in large arteries: steady flow in a carotid bifurcation model. J. Bio-mechanics. 32: 601608

Homma S, Akimoto K, Matsumoto S, Koga J and Tryggvason G 2007 Computations of the breakup of a jet into drops in non-Newtonian liquid-liquid system. J. Chem. Eng. Sci. Japan. 40(11): 920-927

Lakdawala A, Gada V H and Sharma A 2014 A dual grid level set method based study of interfacedynamics for a liquid jet injected upwards into another liquid. Int. J. Multiphase Flow. 59: 206220

Lister J R and Stone H A 1998 Capillary breakup of a viscous thread surrounded by another viscous fluid. Phys. Fluids. 10: 2758-2764 
Mun R P, Byars J A and Boger D V 1998 The effects of polymer concentration and molecular weight on the breakup of laminar capillary jets. J. Non-Newtonian Fluid Mech. 74(1-3): 285-297

Sharma A 2015 Level set method for computational multi-fluid dynamics: A review on developments, applications and analysis. Sadhna - Academy Proc. Eng. Sci. In-Press

Sussman M and Pukett E G 2000 A coupled level set and volume-of-fluid method for computing 3D and axisymmetric incompressible two-phase flows. J. Comp. Phy. 162: 301-337

Sussman M, Smereka P and Osher S 1994 A level set approach for computing solutions to incompressible two-phase flow. J. Comp. Phy. 114: 146

Sussman M, Fatemi E, Smereka P and Osher S 1998 An improved level set method for incompressible two-phase flows. Comput. Fluids 27: 663-680

Song W N and Xia Z M 1994 A phenomenological viscosity model for polymeric fluid. J. Non-Newtonian Fluid Mech. 53(53): 151-163

Tomotika S 1935 On the instability of a cylindrical thread of a viscous liquid surrounded by another viscous fluid. Proc. Roy. Soc. Lond. A 150: 322-337

Yıldırım O E and Basaran O A 2006 Dynamics of formation and dripping of drops of deformation rate thinning and thickening liquids from capillary tubes. J. Non-Newtonian Fluid Mech. 136: 17-37 